\title{
Euler-Poincaré reduction for discrete field theories
}

Joris Vankerschaver

Department of Mathematical Physics and Astronomy

University of Ghent, Krijgslaan 281,

B-9000 Ghent, Belgium

e-mail: Joris.Vankerschaver@UGent.be

\begin{abstract}
In this note, we develop a theory of Euler-Poincaré reduction for discrete Lagrangian field theories. We introduce the concept of Euler-Poincaré equations for discrete field theories, as well as a natural extension of the Moser-Veselov scheme, and show that both are equivalent. The resulting discrete field equations are interpreted in terms of discrete differential geometry. An application to the theory of discrete harmonic mappings is also briefly discussed.
\end{abstract}

MSC classification (2000): 58H05; 65P10; 70S10 


\section{Introduction}

Over the past decades, the interest in discrete mechanics has been steadily increasing. Partly this is due to the development of geometric integrators, which provide a relatively simple means for the long-term numerical integration of mechanical systems. On the other hand, many concepts (Poisson tensors, reduction, etc.) from the continuum theory also have a natural counterpart in the discrete realm, which makes discrete mechanics a subject worthy of interest in its own right. We refer here in particular to the pioneering efforts of Moser and Veselov [17, who studied a class of discrete integrable systems, and Weinstein 21], who was the first to recognize the fact that many of these discrete systems can be understood from the point of view of Lie groupoids.

Inspired by these powerful and elegant methods, a number of people set out to develop a similar geometric approach to classical field theories. Bridges [3] introduced a concept of "multisymplecticity" for Hamiltonian partial differential equations and later Bridges \& Reich [4] studied numerical integrators that conserve a discretized version of multisymplecticity. Independently, Marsden, Patrick, and Shkoller [16] extended the work of Veselov in order to deal with Lagrangian field theories. In a previous paper [20], we built upon their work by extending the Lie-groupoid methods from Weinstein's paper and related work by Marrero, Martín, and Martínez [14 to the case of Lagrangian field theories, allowing us to treat a larger class of field theories, as well as shedding new light on some of the constructions in [16].

In this note, we now focus on a special class of discrete Lagrangian field theories and their behaviour in the presence of symmetry: we introduce a reduction procedure, similar to the one proposed by Marsden, Pekarsky, and Shkoller [15] and Bobenko and Suris [1, 2] for discrete mechanical systems, and show that the resulting discrete field equations have a simple and natural interpretation in the context of discrete geometry.

We begin by introducing a fixed mesh (a certain collection of vertices, edges, and faces) in the space of independent variables. Initially, we are interested in discrete fields that associate to each vertex an element of a given Lie group $G$. If the Lagrangian of such a model is $G$-invariant, we show in $\$ 3$ that these fields can be reduced to a new class of fields that associate a group element to each edge of the given mesh. This reduction procedure is a field-theoretic version of the discrete Euler-Poincaré equations from [15]. We then show that the converse procedure (of reconstruction) is possible only if a certain obstruction vanishes. This obstruction has a natural interpretation as the curvature of a discrete $G$-connection. This is similar to the case of continuum field theories, as studied in [5, 6].

In 4 , we take an alternative route to the Euler-Poincaré equations: inspired by a similar treatment in [5], we prove a discrete version of Noether's theorem and use the $G$-invariance of the Lagrangian to derive the field equations. Finally, in \$5, we consider the Lagrangian of harmonic mappings into a Lie group: we propose an extension of the well-known Moser-Veselov algorithm, demonstrate its equivalence to the Euler-Poincaré 
equations, and establish a particularly clear form of the field equations (involving the concepts of discrete geometry introduced in \$2).

Throughout the paper, a number of simplifying assumptions will be made. In $\S[$ we therefore conclude this paper by giving a brief overview of how these assumptions may be circumvented, and we also indicate how the discrete field theories in this paper fit into the framework of Lie groupoid field theories developed in [20]. In particular, we show that the Euler-Poincaré reduction procedure in $\$ 3$ is just a particular instance of a general reduction theorem in the category of Lie groupoid field theories.

\section{Discrete differential geometry}

\subsection{Discretizing the base space}

In this paper, we consider field theories with two independent variables. Such field theories can be modeled as sections of a fibre bundle with base space $\mathbb{R}^{2}$ (see [16]). In order to discretize these fields, we introduce the set of vertices $V$ in $\mathbb{R}^{2}$ as the set of points in $\mathbb{R}^{2}$ with integer coordinates $(V=\mathbb{Z} \times \mathbb{Z})$. As in finite-difference approximations, the idea is that continuous fields are approximated by specifying their values on the elements of $V$.

For future reference, we also introduce the set of edges $E$, whose elements are ordered pairs of the following form: for all $i, j \in \mathbb{Z} \times \mathbb{Z}$,

$$
((i, j),(i+1, j)) \in E, \quad \text { and } \quad((i, j),(i, j+1)) \in E .
$$

Furthermore, we also demand that if $\left(x_{0}, x_{1}\right) \in E$, then also $\left(x_{1}, x_{0}\right) \in E$, and we write $\left(x_{1}, x_{0}\right)=\left(x_{0}, x_{1}\right)^{-1}$. In other words, $E$ consists of "horizontal" and "vertical" line segments of unit length. To avoid cumbersome notation, we will note an arbitrary element of $E$ as $\mathfrak{e}$, or if we have to refer to its begin and end vertex, as $\left(x_{0}, x_{1}\right)$.

Finally, we introduce the set of faces $F$ as the set of quadruples $\left(\mathfrak{e}_{0}, \mathfrak{e}_{1}, \mathfrak{e}_{2}, \mathfrak{e}_{3}\right)$ of closed paths in $E$, i.e. where the end vertex of $\mathfrak{e}_{i}$ is the begin vertex of $\mathfrak{e}_{i+1}$ for $i=0, \ldots, 3$, and, in addition, we demand that $\mathfrak{e}_{i} \neq \mathfrak{e}_{i+1}^{-1}$ for $i=0, \ldots, 3$. (The indices should be interpreted as being "modulo 4 ": $\mathfrak{e}_{4}$ is just $\mathfrak{e}_{0}$.)

The elements of $F$ can thus be pictured as little squares in $\mathbb{R}^{2}$. A generic element of $F$ will be noted as $\mathfrak{f}$, or, since $\mathfrak{f}$ is fully determined by its bounding vertices, as $\left(x_{0}, x_{1}, x_{2}, x_{3}\right)$. Note that there are two distinct classes of elements in $F$ : those where the vertices are denoted in clockwise and anticlockwise fashion, respectively.

\subsection{Discrete differential forms}

This section is devoted to a review of some elementary concepts of algebraic topology, which will be needed in our discussion of discrete harmonic maps in section 5. Most of 
this section is modeled on [8], as well as on the text book [9].

Consider the mesh $(V, E)$ in $\mathbb{R}^{2}$ introduced in the previous section. The collection of sets $\{V, E, F\}$ together with its various incidence relations determines a cell complex and this leads us naturally to the concepts of homology and cohomology. It is therefore not unreasonable to expect that some of these concepts will enter our study of discrete field theories later on.

An $n$-chain on the given mesh, where $n=0,1,2$, is a formal linear combination (with coefficients in $\mathbb{R}$ ) of " $n$-dimensional elements". More precisely, the vector space of 0 -chains is given by

$$
C_{0}=\left\{\alpha_{1} x_{1}+\cdots+\alpha_{m} x_{m}: \alpha_{1}, \ldots, \alpha_{m} \in \mathbb{R}, x_{1}, \ldots, x_{m} \in V\right\},
$$

where it should be stressed that the elements of $C_{0}$ are formal linear combinations of elements of $V$. Similarly, the vector space $C_{1}$ of 1-chains is generated by elements of $E, C_{2}$ by elements of $F$, and so on. Furthermore, for the space of 1-chains, we adopt the identification that $-\left(x_{0}, x_{1}\right)=\left(x_{1}, x_{0}\right)$.

It is customary to define discrete $n$-forms as $n$-dimensional cochains, i.e. elements of the dual vector space $C_{n}^{*}$ of $C_{n}$. From this definition, it follows immediately that a discrete zero-form induces a function $\phi: V \rightarrow \mathbb{R}$. Conversely, such a function gives rise to a zero-form through linear extension.

Similarly, discrete one-forms can be identified with functions $\varphi$ defined on the set of edges. It should be borne in mind that these functions are not necessarily defined on the whole of $V \times V$, but only on the subset $E$ of edges of the mesh. We also note that $\varphi\left(x_{0}, x_{1}\right)=-\varphi\left(x_{1}, x_{0}\right)$ for any edge $\left(x_{0}, x_{1}\right)$.

We continue by defining discrete two-forms as functions $\psi\left(x_{0}, x_{1}, x_{2}, x_{3}\right)$ on the set of faces. Again, these functions can be extended unambiguously by linearity to a proper cochain. To summarize, we have the following definition:

Definition 2.1. For $n=0,1,2$, a discrete $n$-form is a linear map $f: C_{n} \rightarrow \mathbb{R}$. For $n>2$, all discrete $n$-forms are zero. The set of all discrete $n$-forms is denoted by $C_{n}^{*}$.

For the sake of self-containedness, we recall the explicit form of the coboundary operator $\mathrm{d}: C_{n}^{*} \rightarrow C_{n+1}^{*}$. For a zero-form $\phi, \mathrm{d} \phi\left(x_{0}, x_{1}\right)=\phi\left(x_{1}\right)-\phi\left(x_{0}\right)$. For a one-form $\varphi$,

$$
\mathrm{d} \varphi(\mathfrak{f})=\varphi\left(x_{0}, x_{1}\right)+\varphi\left(x_{1}, x_{2}\right)+\varphi\left(x_{2}, x_{3}\right)+\varphi\left(x_{3}, x_{0}\right) \quad \text { for all } \mathfrak{f}=\left(x_{0}, x_{1}, x_{2}, x_{3}\right) .
$$

The coboundary of a discrete two-form is defined to be zero. We will sometimes refer to $\mathrm{d}$ as the "discrete differential". Note that $\mathrm{d}$ is the dual of the boundary operator $\partial: C_{n+1} \rightarrow C_{n}$, in the sense that $\langle\mathrm{d} f, v\rangle=\langle f, \partial v\rangle$ for all $f \in C_{n}^{*}$ and $v \in C_{n}$, and where $\langle\cdot, \cdot\rangle$ is the natural pairing between $C_{n}$ and $C_{n}^{*}$. This is a discrete analogue of the Stokes theorem. For more information, see [9, p. 186]. 


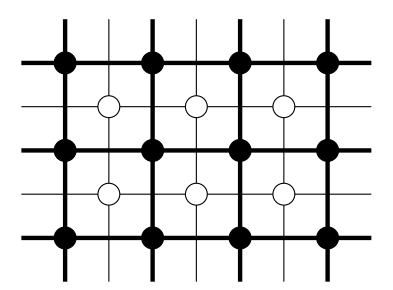

Figure 1: Square mesh (black) and its dual (light).

\subsection{The discrete Hodge star}

In Riemannian geometry, the Hodge star on an oriented manifold $M$ maps $n$-forms on $M$ to $(m-n)$-forms on $M$, where $m=\operatorname{dim} M$. As could be expected from the continuous theory, the discrete Hodge star $\star$, to be introduced below, maps discrete $n$-forms into $(2-n)$-forms. However, there is an additional complication in the discrete case: the forms $\star f$ are not defined on the mesh itself, but rather on a dual mesh, which we now define.

The dual mesh $\left(V^{*}, E^{*}\right)$ is constructed as follows. For every face in $F$, there is a vertex in $V^{*}$ (for which one usually takes the circumcentric dual; see [8]). There is an edge in $E^{*}$ between two vertices $q_{0}, q_{1} \in V^{*}$ if and only if the faces in $F$ corresponding to $q_{0}$ and $q_{1}$ have precisely one edge in common. This determines the sets $V^{*}$ and $E^{*}$; the set $F^{*}$ consists of the faces of this dual graph. It is easy to see that to each face in $F^{*}$, there corresponds a vertex in $V$. See figure 1 for an illustration.

Implicit in this definition is the existence of a duality operator $*$ between $n$-chains on the mesh and $(2-n)$-chains on the dual mesh. This duality is well defined, but only up to orientation; we now use the standard orientation of $\mathbb{R}^{2}$ to settle this point. The definition used here agrees with the algorithm for the orientation of dual cells proposed in [10, remark 2.5.1].

We first define $*$ on the elements of $V, E$, and $F$. By linear extension, it will then be determined on the whole of $C_{n}$. The dual vertex $* \mathfrak{f}$ of a face $\mathfrak{f}$ whose vertices are written down in anticlockwise fashion is the element $r$ of $V^{*}$ introduced above. If, on the other hand, the orientation of $\mathfrak{f}$ is the opposite, then $* \mathfrak{f}$ equals $-r$. We define the dual $* x$ of a vertex $x$ to be the corresponding face in $F^{*}$, with the natural orientation: $* x=\left(r_{1}, \ldots, r_{4}\right)$. Finally, the definition of $*$ on $E$ is slightly more intricate; here, we follow [10]. Let $\left(x_{0}, x_{1}\right)$ be an edge in $E$ and let $\left\{r_{0}, r_{1}\right\}$ be the corresponding dual edge, considered as an unordered set. The line segments $\left[x_{0}, x_{1}\right]$ and $\left[r_{0}, r_{1}\right]$ determine a basis of $\mathbb{R}^{2}$ : if this basis is positively oriented, then $*\left(x_{0}, x_{1}\right)=\left(r_{0}, r_{1}\right)$, otherwise, $*\left(x_{0}, x_{1}\right)=\left(r_{1}, r_{0}\right)$. In the case of the square mesh of figure 11 the action of $*$ on $E$ corresponds to an anticlockwise rotation over $\pi / 2$.

On the dual mesh, one can again introduce discrete forms. We will denote the vector space of discrete $n$-forms on the dual mesh by $D_{n}^{*}$. As the dual mesh is again a square 
mesh, there is a natural way to extend $*$ to an operator from $\left(V^{*}, E^{*}\right)$ to $(V, E)$. It is then easy to check that $* * v=(-1)^{n(2-n)} v$ for any $v \in C_{n}$.

Definition 2.2. The discrete Hodge star $\star: C_{n}^{*} \rightarrow D_{2-n}^{*}$ is defined by

$$
(\star \alpha)(* v)=\alpha(v) \quad \text { for all } v \in C_{n} .
$$

The definition given here is (up to a constant) a special case of the one proposed in [8]. Note that $\star \star \alpha=(-1)^{n(2-n)} \alpha$.

With the discrete Hodge star and the coboundary operator of the previous paragraph, we now arrive at the definition of the discrete codifferential.

Definition 2.3. Let $\alpha$ be a discrete $n$-form. Then the discrete codifferential $\delta \alpha$ is the discrete $(n-1)$-form $\delta \alpha$ defined as $\delta \alpha=\star \mathrm{d} \star \alpha$.

It is useful to write out this definition explicitly for one-forms and two-forms. If $\varphi$ is a discrete one-form, then $\delta \varphi$ is given by

$$
(\delta \varphi)(x)=\varphi\left(x_{1}, x\right)+\varphi\left(x_{2}, x\right)+\varphi\left(x_{3}, x\right)+\varphi\left(x_{4}, x\right),
$$

where $x_{1}, x_{2}, x_{3}, x_{4}$ are the end points of the edges that emanate from $x$. In other words, $\delta \varphi$ assigns to each vertex $x$ the sum of contributions from $\varphi$ on the edges that have $x$ as a vertex. Secondly, for a discrete two-form $\psi$ we note that $\delta \psi$ is the discrete one-form given by $\delta \psi\left(x_{0}, x_{1}\right)=\psi\left(\mathfrak{f}_{0}\right)-\psi\left(\mathfrak{f}_{1}\right)$, where $\mathfrak{f}_{0}$ and $\mathfrak{f}_{1}$ are the faces that have $\left(x_{0}, x_{1}\right)$ as a common edge, and where $\mathfrak{f}_{0}$ is the face where the orientation of the boundary edges agrees with the ordering of $\left(x_{0}, x_{1}\right)$, whereas $\mathfrak{f}_{1}$ is the face with the opposite ordering.

Remark 2.4. Our definition of discrete codifferential agrees with the one from Desbrun et al. 8 up to sign. In their paper, the discrete codifferential is defined as $\delta \alpha=$ $(-1)^{k n+1} \star \mathrm{d} \star \alpha$, where $k$ is the dimension of the ambient space. Note that here, as $k=2,(-1)^{k n+1}=-1$ regardless of $n$.

\subsection{Discrete connections}

In the preceding sections, we introduced discrete one-forms as assignments of a real number to each edge $\mathfrak{e} \in E$. This theory can be extended in a straightforward way to discrete forms taking values in an arbitrary Abelian Lie group $G$, the only significant difference being that we have to redefine the spaces of $n$-chains $C_{n}$ as consisting of formal linear combinations with coefficients in $\mathbb{Z}$.

For instance, if $\varphi: C_{1} \rightarrow G$ is a discrete one-form, then $\mathrm{d} \varphi$ is determined by its action on the set $F$ by (2) and can be extended by linearity to yield a map from $C_{2}$ to $G$, where it should be borne in mind that the elements of $C_{2}$ are still formal linear combinations of elements in $F$, but now with coefficients in $\mathbb{Z}$. 
The theory of discrete forms with values in an Abelian Lie group will be used in section 5 , but in the general case, we will be confronted with mappings from $E$ to a non-Abelian Lie group $G$. Such maps can no longer be interpreted as discrete one-forms. Luckily, it turns out that these maps have a natural interpretation as discrete G-connections, which we now define.

Definition 2.5. A discrete $G$-connection is a map $\omega: E \rightarrow G$, such that, for all edges $\mathfrak{e} \in E, \omega\left(\mathfrak{e}^{-1}\right)=\omega(\mathfrak{e})^{-1}$. The curvature of such a connection is the map $\Omega: F \rightarrow G$ defined as $\Omega(\mathfrak{f})=\omega\left(\mathfrak{e}_{1}\right) \cdots \omega\left(\mathfrak{e}_{4}\right)$, where $\mathfrak{e}_{1}, \ldots, \mathfrak{e}_{4}$ are the boundary edges of the face $\mathfrak{f}$. A discrete $G$-connection is said to be flat if $\Omega(\mathfrak{f})=e$ for all $\mathfrak{f} \in F$.

Note that in the case of a non-flat connection, $\Omega(\mathfrak{f})$ depends not only on $\mathfrak{f}$, but also on the exact representation of $\mathfrak{f}$ as a set of edges $\mathfrak{e}_{1}, \ldots, \mathfrak{e}_{4}$ (any cyclic permutation of this set represents the same face). However, this indeterminacy does not occur for flat connections, the only case that we will consider later on.

The theory of discrete $G$-connections closely mimics the usual theory of connections. As an example, we mention the following proposition, from which a number of interesting properties may be deduced.

Proposition 2.6. Consider a discrete $G$-connection $\omega: E \rightarrow G$. If $\omega$ is flat, then there exists a unique mapping $\phi: V \times V \rightarrow G$ such that $\phi_{\mid E}=\omega$.

This follows immediately from [20, prop. 7], or can be proved directly as follows.

We define a path in $E$ to be a sequence $\mathfrak{e}_{1}, \mathfrak{e}_{2}, \ldots, \mathfrak{e}_{m}$ of edges, such that the end vertex of $\mathfrak{e}_{i}$ is the begin vertex of $\mathfrak{e}_{i+1}$ (for $i=1, \ldots, m-1$ ). Let $\omega: E \rightarrow G$ be any discrete $G$-connection. In particular, $\omega$ need not be flat. Then, $\omega$ induces a map $\hat{\omega}$ from the set of paths to $G$ as follows:

$$
\hat{\omega}\left(\mathfrak{e}_{1}, \mathfrak{e}_{2}, \ldots, \mathfrak{e}_{m}\right)=\omega\left(\mathfrak{e}_{1}\right) \omega\left(\mathfrak{e}_{2}\right) \cdots \omega\left(\mathfrak{e}_{m}\right)
$$

We call $\hat{\omega}$ the "discrete holonomy mapping". Note that if $\omega$ is a flat connection, one can easily prove that $\hat{\omega}$ maps closed paths to the unit in $G$. Furthermore, in the case of a flat connection, it can be easily established that simplicially homotopic paths have the same image under $\hat{\omega}$. (This is the discrete counterpart of a well-known theorem of continuous connections (see [11, p. 93]): if $\omega$ is a flat connection, then any two closed homotopic loops have the same holonomy.)

Let us now return to the discrete theory. In the case of a flat connection on a simplyconnected cell complex, we have seen that $\hat{\omega}$ does not depend on the choice of path between two fixed points, and induces therefore a map $\hat{\omega}: V \times V \rightarrow G$. This map satisfies the requirements in proposition 2.6.

Remark 2.7. The concept of discrete $G$-connections used here is common in lattice gauge theories (see [22]). Novikov [18] studied a similar concept. 
Closely related to this definition is the concept of discrete connection on a discrete principal fibre bundle (see [12] for definitions and [14, §5.4] for an application to discrete reduction). The latter could be used to extend the results in this paper to the case of Lagrange-Poincaré reduction, where a $G$-invariant Lagrangian on a pair groupoid $Q \times Q$ is given. If $Q$ coincides with the symmetry group $G$, then Lagrange-Poincaré reduction is just Euler-Poincaré reduction, the case considered here.

\section{Discrete Euler-Poincaré reduction}

In this section, we begin our study of discrete Lagrangian field theories. Initially, we define discrete fields as follows:

Definition 3.1. A discrete field is a map $\phi: V \rightarrow G$.

If the discrete Lagrangian, to be defined below, is $G$-invariant, we shall see that these fields induce a new class of discrete fields, that associate a group element to each edge. From the last section, we know that such maps have a natural interpretation as discrete G-connections.

In theorem [3.4, it is shown how the field equations for the unreduced fields $\phi$ are equivalent to a set of equations, called discrete Euler-Poincaré equations, for the reduced fields $\varphi$. Both sets of equations arise by extremizing a certain action functional. In theorem 3.6, we deal with the reconstruction problem. Starting from a reduced field $\varphi: E \rightarrow G$, it is shown that $\varphi$ gives rise to a solution $\phi: V \rightarrow G$ of the original field equations if and only if the curvature of $\varphi$ vanishes. This treatment was inspired by the work of Castrillón and Ratiu [6], who developed Lagrangian reduction for field theories in the continuous case.

\subsection{The discrete Lagrangian}

Definition 3.2. A discrete Lagrangian is a function $L: G \times G \times G \rightarrow \mathbb{R}$.

A word of explanation is in order here. In the continuous case, a Lagrangian is a function $\mathcal{L}: J^{1} \pi \rightarrow \mathbb{R}$, where $J^{1} \pi$ is the first jet bundle of the bundle $\pi: \mathbb{R}^{2} \times G \rightarrow \mathbb{R}^{2}$. It can be shown that, in this case, $J^{1} \pi$ is isomorphic to $\mathbb{R}^{2} \times(T G \oplus T G)$ (see [19, lemma 4.1.20]). Under the (modest) assumption that Lagrangian $\mathcal{L}$ does not depend on the coordinates of the base space, we therefore conclude that $\mathcal{L}$ is just a function on $T G \oplus T G$.

The idea of defining a discrete Lagrangian as a function on $G \times G \times G$ then follows from the idea of Moser and Veselov of approximating $T G$ by $G \times G$ : by applying this Veselov-type discretization twice (once in the "horizontal" and once in the "vertical" 


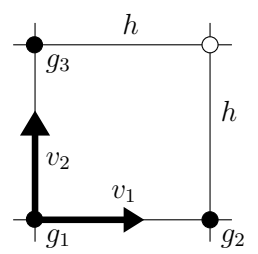

Figure 2: Discretization of $T G \oplus T G$ by $G \times G \times G$

direction of the square mesh), it follows that $T G \oplus T G$ has $G \times G \times G$ as a natural discrete counterpart; see figure 2.

The Lie group $G$ has a natural diagonal action by left translations on the Cartesian product $G \times G: g \cdot\left(g_{1}, g_{2}\right)=\left(g g_{1}, g g_{2}\right)$. We now wish to study the situation where $L$ is invariant under this action. As the quotient $(G \times G) / G$ is naturally isomorphic to the Lie group $G$ itself, a discrete field $\phi: V \rightarrow G$ induces a "reduced field" $\varphi: E \rightarrow G$ as follows:

$$
\varphi(\mathfrak{e})=\phi\left(x_{0}\right)^{-1} \phi\left(x_{1}\right), \quad \text { for all } \mathfrak{e}=\left(x_{0}, x_{1}\right) \in E .
$$

This leads us to the following definition:

Definition 3.3. A reduced discrete field is a map $\varphi: E \rightarrow G$ such that $\varphi\left(\mathfrak{e}^{-1}\right)=$ $\varphi(\mathfrak{e})^{-1}$. Equivalently, it is a discrete $G$-connection.

The reduced field $\varphi$ induced by a discrete field $\phi: V \rightarrow G$ is flat when considered as a discrete $G$-connection. Hence, by proposition 2.6 (or simply by construction), it may be extended to a map $\varphi: V \times V \rightarrow G$. However, not all reduced fields are flat.

The Lagrangian $L$ gives rise to a reduced Lagrangian $l: G \times G \rightarrow \mathbb{R}$ defined by

$$
l\left(g_{1}^{-1} g_{2}, g_{1}^{-1} g_{3}\right)=L\left(g_{1}, g_{2}, g_{3}\right) .
$$

Let $\Phi: G \times G \rightarrow G$ be the map defined as $\Phi\left(g, g^{\prime}\right)=g^{-1} g^{\prime}$, so that the reduced field $\varphi$ associated to a discrete field $\phi$ is given by

$$
\varphi=\Phi \circ(\phi \times \phi)
$$

Furthermore, we introduce the following mapping:

$$
\hat{\Psi}:\left(g_{1}, g_{2}, g_{3}\right) \in G \times G \times G \mapsto\left(g_{1}^{-1} g_{2}, g_{1}^{-1} g_{3}\right) \in G \times G .
$$

It is obvious that the Lagrangian $L$ is related to the reduced Lagrangian by $L=l \circ \hat{\Psi}$. For future reference, we also introduce a map $\bar{\Psi}: T\left(G^{3}\right) \rightarrow(G \times \mathfrak{g})^{3}$ as

$$
\bar{\Psi}\left(v_{1}, v_{2}, v_{3}\right)=\left(g_{1}, g_{2}, g_{3} ; T L_{g_{1}^{-1}}\left(v_{1}\right), T L_{g_{2}^{-1}}\left(v_{2}\right), T L_{g_{3}^{-1}}\left(v_{3}\right)\right)
$$

where $v_{i} \in T_{g_{i}} G$. 
An unreduced field $\phi: V \rightarrow G$ can be interpreted as an assignment of an element $\phi_{i, j}$ in $G$ to each vertex $(i, j)$. Similarly, a reduced field $\varphi: E \rightarrow G$ can be described as an assignment of a group element $u_{i, j}$ to each "vertical" edge $((i, j+1),(i, j))$, and of a group element $v_{i, j}$ to each "horizontal" edge $((i, j),(i+1, j))$, where

$$
u_{i, j}=\phi_{i, j}^{-1} \phi_{i+1, j} \quad \text { and } \quad v_{i, j}=\phi_{i, j}^{-1} \phi_{i, j+1} .
$$

We will use these notations for the remainder of the paper.

\subsection{The reduction problem}

Given a discrete Lagrangian $L$, the discrete action sum $S$ is given by

$$
S(\phi)=\sum_{(i, j) \in U} L\left(\phi_{i, j}, \phi_{i+1, j}, \phi_{i, j+1}\right),
$$

where $\phi$ is a map from $V$ to $G$, and where $U$ is a finite subset of the set of vertices $V$. In [16], it was shown that $\phi$ is an extremum of this action if and only $\phi$ satisfies the following set of discrete Euler-Lagrange equations: for all $(i, j) \in V$,

$$
\frac{\partial L}{\partial g_{1}}\left(\phi_{i, j}, \phi_{i+1, j}, \phi_{i, j+1}\right)+\frac{\partial L}{\partial g_{2}}\left(\phi_{i-1, j}, \phi_{i, j}, \phi_{i-1, j+1}\right)+\frac{\partial L}{\partial g_{3}}\left(\phi_{i, j-1}, \phi_{i+1, j-1}, \phi_{i, j}\right)=0 .
$$

Similarly, we may define the reduced action sum $s$ as

$$
s(\varphi)=\sum_{(i, j) \in U} l\left(u_{i, j}, v_{i, j}\right) .
$$

A reduced field $\varphi: E \rightarrow G$ is an extremum of $s$ if and only if it satisfies the discrete Euler-Poincaré equations, to be derived below. The central aspects of discrete EulerPoincaré reduction are summarized in the following theorem. This theorem, as well as its proof, are very similar to the discrete reduction process in mechanics (see [15]).

Theorem 3.4 (Reduction). Let $L$ be a $G$-invariant Lagrangian on $G \times G \times G$ and consider the reduced Lagrangian $l$ on $G \times G$. Consider a discrete field $\phi: V \rightarrow G$ and let $\varphi: V \times V \rightarrow G$ be the induced reduced field. Then the following are equivalent:

(a) $\phi$ is a solution of the discrete Euler-Lagrange equations for L;

(b) $\phi$ is an extremum of the action sum $S$ for arbitrary variations;

(c) the reduced field $\varphi$ is a solution of the discrete Euler-Poincaré equations:

$$
\begin{aligned}
& {\left[\left(R_{u_{i, j}}^{*} \mathrm{~d} l\left(\cdot, v_{i, j}\right)\right)_{e}-\left(L_{u_{i-1, j}}^{*} \mathrm{~d} l\left(\cdot, v_{i-1, j}\right)\right)_{e}\right]+} \\
& {\left[\left(R_{v_{i, j}}^{*} \mathrm{~d} l\left(u_{i, j}, \cdot\right)\right)_{e}-\left(L_{v_{i, j-1}}^{*} \mathrm{~d} l\left(u_{i, j-1}, \cdot\right)\right)_{e}\right]=0 ;}
\end{aligned}
$$


(d) the reduced field $\varphi$ is an extremum of the reduced action sum $s$ for variations of the form

$$
\delta u_{i, j}=T L_{u_{i, j}}\left(\theta_{i+1, j}\right)-T R_{u_{i, j}}\left(\theta_{i, j}\right) \in T_{u_{i, j}} G
$$

and

$$
\delta v_{i, j}=T L_{v_{i, j}}\left(\theta_{i, j+1}\right)-T R_{v_{i, j}}\left(\theta_{i, j}\right) \in T_{v_{i, j}} G,
$$

where $\theta_{i, j}=T L_{\phi_{i, j}^{-1}}\left(\delta \phi_{i, j}\right) \in \mathfrak{g}$.

Proof. The equivalence of (国) and (b) follows from a standard argument in discrete Lagrangian field theories, and was shown in [20].

In order to prove the equivalence of (B) and (d) , we note that $L=l \circ \hat{\Psi}$ (see (44)), from which we conclude that if $\varphi=\Phi \circ \phi$, then $S(\phi)=s(\varphi)$. Now, consider the components $\left\{u_{i, j}\right\}$ and $\left\{v_{i, j}\right\}$ of the reduced field, as in ([6). It is easy to check that an arbitrary variation $\epsilon \mapsto \phi_{i, j}(\epsilon)$ of $\phi$ induces corresponding variations $\delta u_{i, j}$ and $\delta v_{i, j}$ of $u_{i, j}$ and $v_{i, j}$, given by (9) and (10).

Finally, we show the equivalence of (did) with the Euler-Poincaré equations (지). From the reduced action sum $s(\varphi)$ we obtain that

$$
\begin{aligned}
\left.\frac{\mathrm{d}}{\mathrm{d} \epsilon} s(\varphi(\epsilon))\right|_{\epsilon=0} & =\left.\sum_{i, j} \frac{\mathrm{d}}{\mathrm{d} \epsilon} l\left(u_{i, j}(\epsilon), v_{i, j}(\epsilon)\right)\right|_{\epsilon=0} \\
& =\sum_{i, j}\left(\mathrm{~d} l\left(\cdot, v_{i, j}\right) \cdot \delta u_{i, j}+\mathrm{d} l\left(u_{i, j}, \cdot\right) \cdot \delta v_{i, j}\right) .
\end{aligned}
$$

Substitution of (91) and (10) into this expression then yields (after relabelling some of the summation indices) the discrete Euler-Poincaré equations (8).

In this context, a variation can be interpreted in two ways. In the case of unreduced fields, a variation of a field $\phi_{i, j}$ is a map $\delta \phi: V \rightarrow T G$ such that $\delta \phi_{i, j} \in T_{\phi_{i, j}} G$. In the case of reduced fields, a variation of a field $\left\{u_{i, j}, v_{i, j}\right\}$ is a pair of maps $\delta u, \delta v: V \rightarrow T G$ such that $\delta u_{i, j} \in T_{u_{i, j}} G$ and $\delta v_{i, j} \in T_{v_{i, j}} G$. In both cases, we demand that the variation is zero on the boundary of $U$ :

$$
\delta \phi_{i, j}=0, \quad \text { and } \quad \delta u_{i, j}=\delta v_{i, j}=0 \quad \text { for all }(i, j) \in \partial U .
$$

Here, the boundary $\partial U$ is defined as the set of vertices $(i, j)$ such that $(i, j)$ is a vertex of at least one face in $U$, and at least one face not in $U$ (see [16]).

\subsection{The reconstruction problem}

From theorem 3.4. we know that a solution $\phi: V \rightarrow G$ of the discrete Euler-Lagrange equations gives rise to a reduced field $\varphi: E \rightarrow G$, which has a natural interpretation as a flat discrete connection in the sense of definition 2.5 
To tackle the converse problem, we use the following consequence of proposition 2.6. Recall that the holonomy mapping $\hat{\omega}$ of a flat discrete connection $\omega$ is a map from $V \times V$ to $G$.

Proposition 3.5. Let $\omega$ be a flat discrete $G$-connection with associated discrete holonomy $\hat{\omega}: V \times V \rightarrow G$. Then there exists a map $\phi: V \rightarrow G$ such that $\omega\left(x_{0}, x_{1}\right)=$ $\phi\left(x_{0}\right)^{-1} \phi\left(x_{1}\right)$. The map $\phi$ is unique up to left translation by an element of $G$.

Proof. Choose an arbitrary vertex $x_{0}$ and a group element $g_{0}$, and define $\phi\left(x_{0}\right)=g_{0}$. Let $x_{1}$ be any other vertex and put $\phi\left(x_{1}\right)=g_{0} \hat{\omega}\left(x_{0}, x_{1}\right)$. This map is well defined.

Let $\varphi: E \rightarrow G$ be a solution of the discrete Euler-Poincaré equations (8). We now wish to reconstruct a solution $\phi$ of the original problem, such that $\varphi=\Phi \circ \phi$. The map $\phi$ is provided by proposition 3.5, on the condition that $\varphi$ is a flat connection. As soon as $\varphi$ is not flat, the holonomy $\hat{\omega}$ is path dependent, and no such $\phi$ can exist. Therefore, we have the following theorem.

Theorem 3.6 (reconstruction). Let $\varphi: E \rightarrow G$ be a solution of the discrete EulerPoincaré equations (8). There exists a solution $\phi: V \rightarrow G$ of the unreduced EulerLagrange equations (7) if and only if $\varphi$ is flat. In that case, $\phi$ is uniquely determined up to left translation by an element of $G$.

Remark 3.7. In some cases, the element $g_{0} \in G$ used in constructing the map $\phi$ is fixed by considering boundary or initial conditions on the solutions.

\section{The Noether theorem}

In the case of continuous field theories, Noether's theorem states that, for each continuous symmetry, there exists a conservation law (see [7] for an overview). Here, we will show that a similar theorem holds for discrete field theories with a continuous symmetry, and that the conservation law associated to the left $G$-invariance of the Lagrangian is equivalent to the Euler-Poincaré equations. A similar theorem was proved in [5] in the case of Lagrangian reduction for continuous field theories.

\subsection{The Poincaré-Cartan forms}

We begin by introducing a set of Poincaré-Cartan forms that will be instrumental in formulating the Noether theorem. It should be remarked that the definition of the Poincaré-Cartan forms here is a special case of a more general construction, elaborated in [20] and briefly outlined in section 6.

As in section 3, let $L: G^{3}=G \times G \times G \rightarrow \mathbb{R}$ be a $G$-invariant Lagrangian and consider the reduced Lagrangian $l: G^{2}=G \times G \rightarrow \mathbb{R}$. Associated to $L$ is a set of three one-forms, 
called Poincaré-Cartan forms, and defined as follows:

$$
\theta_{(1)}^{L}: G^{3} \rightarrow T^{*}\left(G^{3}\right), \quad\left\langle\theta_{(1)}^{L}\left(g_{1}, g_{2}, g_{3}\right),\left(v_{1}, v_{2}, v_{3}\right)\right\rangle=\left\langle\mathrm{d} L\left(\cdot, g_{2}, g_{3}\right)_{g_{1}}, v_{1}\right\rangle
$$

with $v_{i} \in T_{g_{i}} G$, and similarly for $\theta_{(2)}^{L}$ and $\theta_{(3)}^{L}$. Note that $\theta_{(1)}^{L}+\theta_{(2)}^{L}+\theta_{(3)}^{L}=\mathrm{d} L$.

For the reduced Lagrangian $l$, the definition of the Poincaré-Cartan forms is somewhat less direct. We put

$$
\begin{gathered}
\theta_{(1)}^{l}: G^{2} \rightarrow \mathfrak{g}^{*} \oplus \mathfrak{g}^{*} \oplus \mathfrak{g}^{*}, \\
\left\langle\theta_{(1)}^{l}(u, v),\left(\xi_{1}, \xi_{2}, \xi_{3}\right)\right\rangle=-\left\langle\mathrm{d} l(\cdot, v), T R_{u}\left(\xi_{1}\right)\right\rangle-\left\langle\mathrm{d} l(u, \cdot), T R_{v}\left(\xi_{1}\right)\right\rangle,
\end{gathered}
$$

where $\xi_{1}, \xi_{2}, \xi_{3} \in \mathfrak{g}$. Alternatively, if $t \mapsto h(t)$ is a curve in $G$ such that $h(0)=e$ and $\dot{h}(0)=\xi_{1}, \theta_{(1)}^{l}$ may be defined as

$$
\left\langle\theta_{(1)}^{l}(u, v),\left(\xi_{1}, \xi_{2}, \xi_{3}\right)\right\rangle=\left.\frac{\mathrm{d}}{\mathrm{d} t} l\left(h(t)^{-1} u, h(t)^{-1} v\right)\right|_{t=0} .
$$

The remaining one-forms $\theta_{(2)}^{l}$ and $\theta_{(3)}^{l}$ are defined by

$$
\left\langle\theta_{(2)}^{l}(u, v),\left(\xi_{1}, \xi_{2}, \xi_{3}\right)\right\rangle=\left\langle\mathrm{d} l(\cdot, v), T L_{u}\left(\xi_{2}\right)\right\rangle
$$

and

$$
\left\langle\theta_{(3)}^{l}(u, v),\left(\xi_{1}, \xi_{2}, \xi_{3}\right)\right\rangle=\left\langle\mathrm{d} l(u, \cdot), T L_{v}\left(\xi_{3}\right)\right\rangle .
$$

The apparent asymmetry between (12) and (13, 14) is due to our definition of $l$. For a fully symmetric set of Poincaré-Cartan forms, one should follow the prescriptions of 20 .

The Lagrangian $L$ is related to the reduced Lagrangian $l$ as follows: $L=l \circ \hat{\Psi}$. A straightforward computation (or the application of theorem 25 in [20]) shows us that the corresponding Poincaré-Cartan forms are related in a similar way:

Lemma 4.1. Let $\Psi$ be the bundle map $(\hat{\Psi}, \bar{\Psi})$ as defined above. Then

$$
\Psi^{\star} \theta_{(i)}^{l}=\theta_{(i)}^{L} .
$$

Furthermore, a similar identity holds for the presymplectic forms $\Omega_{(i)}^{l}:=-\mathrm{d} \theta_{(i)}^{l}$ and $\Omega_{(i)}^{L}:=-\mathrm{d} \theta_{(i)}^{L}$ :

$$
\Psi^{\star} \Omega_{(i)}^{l}=\Omega_{(i)}^{L}
$$

We recall that the pullback of $\theta_{(i)}^{l}$ by the bundle map $\Psi=(\hat{\Psi}, \bar{\Psi}): T(G)^{3} \rightarrow(G \times \mathfrak{g})^{3}$ is defined as follows:

$$
\left\langle\left(\Psi^{\star} \theta_{(i)}^{l}\right)\left(g_{1}, g_{2}, g_{3}\right),\left(v_{1}, v_{2}, v_{3}\right)\right\rangle=\left\langle\theta_{(i)}^{l}\left(\hat{\Psi}\left(g_{1}, g_{2}, g_{3}\right)\right), \bar{\Psi}\left(v_{1}, v_{2}, v_{3}\right)\right\rangle .
$$




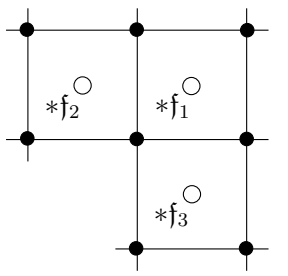

Figure 3: Location of $\mathfrak{f}_{1}, \mathfrak{f}_{2}$, and $\mathfrak{f}_{3}$.

\subsection{The unreduced Lagrangian}

The Lagrangian $L: G^{3} \rightarrow \mathbb{R}$ is assumed to be left $G$-invariant in the sense that $L\left(g g_{1}, g g_{2}, g g_{3}\right)=L\left(g_{1}, g_{2}, g_{3}\right)$ for all $g$ in $G$. According to Noether's theorem, which we will prove in a moment, there is a conservation law associated to this symmetry.

Let $\xi$ be an element of $\mathfrak{g}$. Infinitesimal invariance of Lagrangian under the flow generated by $\xi$ is expressed as

$$
\left\langle\mathrm{d} L\left(g_{1}, g_{2}, g_{3}\right),\left(\xi_{G}\left(g_{1}\right), \xi_{G}\left(g_{2}\right), \xi_{G}\left(g_{3}\right)\right)\right\rangle=0,
$$

where $\xi_{G}$, defined by $\xi_{G}(g)=T R_{g}(\xi)$, is the fundamental vector field associated to $\xi$. Consider the functions $J_{\xi}^{i}: G^{3} \rightarrow \mathbb{R}, i=1,2,3$, given by

$$
J_{\xi}^{i}\left(g_{1}, g_{2}, g_{3}\right)=\left\langle\theta_{(i)}^{L}\left(g_{1}, g_{2}, g_{3}\right),\left(\xi_{G}\left(g_{1}\right), \xi_{G}\left(g_{2}\right), \xi_{G}\left(g_{3}\right)\right)\right\rangle
$$

As $J_{\xi}^{i}$ is linear in $\xi$, we can define a map $J^{i}: G^{3} \rightarrow \mathfrak{g}^{*}$ by the prescription $\left\langle J^{i}, \xi\right\rangle=J_{\xi}^{i}$, for all $\xi \in \mathfrak{g}$. An immediate consequence of the $G$-invariance is that

$$
J^{1}+J^{2}+J^{3}=0
$$

Now, let there be given a map $\phi: V \rightarrow G$ and consider the pull-back of each $J^{i}$ by $\psi:=\phi \times \phi \times \phi$. In this way, we obtain a map from $F$ to $\mathfrak{g}^{*}$, which can be identified, by means of the discrete Hodge star, with a map from $V^{*}$ to $\mathfrak{g}^{*}$. In view of (16) it turns out that we only need to consider two such maps, which we will denote by $\eta^{(x)}$ and $\eta^{(y)}$, and which are given by

$$
\eta^{(x)}(r)=J^{2}\left(\phi\left(x_{1}\right), \phi\left(x_{2}\right), \phi\left(x_{3}\right)\right), \quad \text { and } \quad \eta^{(y)}(r)=J^{3}\left(\phi\left(x_{1}\right), \phi\left(x_{2}\right), \phi\left(x_{3}\right)\right)
$$

for $r \in V^{*}$, and where $\mathfrak{f}=\left(x_{1}, x_{2}, x_{3}, x_{4}\right)$ is the face dual to $r: * \mathfrak{f}=r$. We further put $\eta_{\xi}^{(x)}=\left\langle\eta^{(x)}, \xi\right\rangle$ and $\eta_{\xi}^{(y)}=\left\langle\eta^{(y)}, \xi\right\rangle$.

Before stating Noether's theorem, we recall the definition of the backward difference operators $\delta_{x}^{-}$and $\delta_{y}^{-}$: if $r_{1}, r_{2}$ and $r_{3}$, with $r_{i}=* \mathfrak{f}_{i}$, are located as in figure 3, and $f: V^{*} \rightarrow \mathbb{R}$ is a function, then

$$
\left(\delta_{x}^{-} f\right)\left(r_{1}\right)=f\left(r_{1}\right)-f\left(r_{2}\right) \quad \text { and } \quad\left(\delta_{y}^{-} f\right)\left(r_{1}\right)=f\left(r_{1}\right)-f\left(r_{3}\right)
$$


These difference operators can be extended without difficulty to the case of vectorvalued functions.

Proposition 4.2 (Noether). Consider a G-invariant Lagrangian $L: G^{3} \rightarrow \mathbb{R}$. If $\phi$ is a solution of the discrete field equations (7), then the maps $\eta^{(x)}, \eta^{(y)}: V^{*} \rightarrow \mathfrak{g}^{*}$ satisfy the following conservation law:

$$
\delta_{x}^{-} \eta^{(x)}+\delta_{y}^{-} \eta^{(y)}=0
$$

Conversely, if $\phi$ is such that the associated mappings $\eta^{(x)}$ and $\eta^{(y)}$ satisfy (17), then $\phi$ is a solution of the discrete field equations.

Proof. Consider points $r_{1}, r_{2}, r_{3}$ of the dual mesh as in figure 3 and let $\mathfrak{f}_{i}$ be the dual face to $r_{i}: \mathfrak{f}_{i}=* r_{i}$. Then we have that

$$
\begin{aligned}
\delta_{x}^{-} \eta_{\xi}^{(x)}\left(r_{1}\right)+\delta_{y}^{-} \eta_{\xi}^{(y)}\left(r_{1}\right) & =\eta_{\xi}^{(x)}\left(r_{1}\right)-\eta_{\xi}^{(x)}\left(r_{2}\right)+\eta_{\xi}^{(y)}\left(r_{1}\right)-\eta_{\xi}^{(y)}\left(r_{3}\right) \\
& =J_{\xi}^{2}\left(\psi\left(\mathfrak{f}_{1}\right)\right)-J_{\xi}^{2}\left(\psi\left(\mathfrak{f}_{2}\right)\right)+J_{\xi}^{3}\left(\psi\left(\mathfrak{f}_{1}\right)\right)-J_{\xi}^{3}\left(\psi\left(\mathfrak{f}_{3}\right)\right) \\
& =-J_{\xi}^{1}\left(\psi\left(\mathfrak{f}_{1}\right)\right)-J_{\xi}^{2}\left(\psi\left(\mathfrak{f}_{2}\right)\right)-J_{\xi}^{3}\left(\psi\left(\mathfrak{f}_{3}\right)\right),
\end{aligned}
$$

where we have used (16). Recalling that $\psi$ is defined as $\psi(\mathfrak{f})=\left(\phi\left(x_{1}\right), \phi\left(x_{2}\right), \phi\left(x_{3}\right)\right)$, we note that

$$
J_{\xi}^{i}(\psi(\mathfrak{f}))=\theta_{(i)}^{L}(\psi(\mathfrak{f}))\left(\xi_{G}, \xi_{G}, \xi_{G}\right)=\left\langle\frac{\partial L}{\partial g_{i}}(\psi(\mathfrak{f})), \xi_{G}\right\rangle .
$$

Therefore, we can rewrite the conservation law (18) as

$$
\delta_{x}^{-} \eta_{\xi}^{(x)}\left(r_{1}\right)+\delta_{y}^{-} \eta_{\xi}^{(y)}\left(r_{1}\right)=-\left\langle\frac{\partial L}{\partial g_{1}}\left(\psi\left(\mathfrak{f}_{1}\right)\right)+\frac{\partial L}{\partial g_{2}}\left(\psi\left(\mathfrak{f}_{2}\right)\right)+\frac{\partial L}{\partial g_{3}}\left(\psi\left(\mathfrak{f}_{3}\right)\right), \xi_{G}\right\rangle,
$$

which vanishes if $\phi$ satisfies the field equations. Conversely, if the left hand side is zero, then $\phi$ is a solution of the field equations.

\subsection{The reduced Lagrangian}

Not only is Noether's theorem equivalent to the unreduced discrete field equations, it will turn out that it contains the Euler-Poincaré equations as well. To show this, we start from the discrete conservation law as expressed by (18). We use the same notational conventions as in the preceding section and write

$$
\hat{\Psi}\left(\psi\left(\mathfrak{f}_{i}\right)\right)=\left(u_{i}, v_{i}\right), \quad i=1,2,3 .
$$

Furthermore, we note that $\left(\psi\left(\mathfrak{f}_{1}\right)\right)_{1}=\left(\psi\left(\mathfrak{f}_{2}\right)\right)_{2}=\left(\psi\left(\mathfrak{f}_{3}\right)\right)_{3}$; this unique element of $G$ is denoted by $g$. By rewriting each of the three expressions in (18) in terms of the reduced Lagrangian $l$ only, we obtain

$$
J_{\xi}^{1}\left(\psi\left(\mathfrak{f}_{1}\right)\right)=-\left\langle R_{u_{1}}^{*} \mathrm{~d} l\left(\cdot, v_{1}\right), \eta\right\rangle-\left\langle R_{v_{1}}^{*} \mathrm{~d} l\left(u_{1}, \cdot\right), \eta\right\rangle,
$$


where $\eta=\operatorname{Ad}_{g^{-1}} \xi$, as well as

$$
J_{\xi}^{2}\left(\psi\left(\mathfrak{f}_{2}\right)\right)=\left\langle L_{u_{2}}^{*} \mathrm{~d} l\left(\cdot, v_{2}\right), \eta\right\rangle \quad \text { and } \quad J_{\xi}^{3}\left(\psi\left(\mathfrak{f}_{3}\right)\right)=\left\langle L_{v_{3}}^{*} \mathrm{~d} l\left(u_{3}, \cdot\right), \eta\right\rangle .
$$

Putting all of these expressions together gives the following:

$$
\begin{aligned}
\delta_{x}^{-} \eta_{\xi}^{(x)}\left(r_{1}\right)+\delta_{y}^{-} \eta_{\xi}^{(y)}\left(r_{1}\right)=\left\langle\left[R_{u_{1}}^{*} \mathrm{~d} l\left(\cdot, v_{1}\right)-L_{u_{2}}^{*} \mathrm{~d} l\left(\cdot, v_{2}\right)\right]+\right. & \\
& {\left.\left[R_{v_{1}}^{*} \mathrm{~d} l\left(u_{1}, \cdot\right)-L_{v_{3}}^{*} \mathrm{~d} l\left(u_{3}, \cdot\right)\right], \eta\right\rangle . }
\end{aligned}
$$

As $\eta$ ranges over the whole of $\mathfrak{g}$, we conclude that the conservation law (17) implies the discrete Euler-Poincaré equation (묘).

\section{Extending the Moser-Veselov approach}

In their seminal paper, Moser and Veselov [17] approached the problem of finding an integrable discretization of the rigid-body equations by embedding the group $S O(n)$ into a linear space, namely $\mathfrak{g l}(n)$. Somewhat later, Marsden, Pekarsky, and Shkoller 15 then developed a general procedure of Lagrangian reduction for discrete mechanical systems, and showed that the Moser-Veselov equations are equivalent to the discrete Lie-Poisson equations.

Here, we intend to do the same thing for a fundamental model in field theory: that of harmonic mappings from $\mathbb{R}^{2}$ into a Lie group $G$. We will show that it is possible to develop a Moser-Veselov type discretization of these field equations, provided that $G$ is embedded in a linear space. As could be expected, these discrete field equations are equivalent to the Euler-Poincaré equations.

In the continuous case, the harmonic mapping Lagrangian is given by

$$
\mathcal{L}=\frac{1}{2}\left\langle\phi^{-1} \phi_{x}, \phi^{-1} \phi_{x}\right\rangle+\frac{1}{2}\left\langle\phi^{-1} \phi_{y}, \phi^{-1} \phi_{y}\right\rangle
$$

where $\langle\cdot, \cdot\rangle$ is the Killing form on $\mathfrak{g}$ and where the subscript ' $x$ ' and ' $y$ ' denote partial differentiation with respect to that variable. For the sake of clarity, we will only treat the case of harmonic maps that take values in $S O(n)$, embedded in $\mathfrak{g l}(n)$, in which case the Killing form is just the trace. We stress that the entire theory can be generalized to the case of an arbitrary semi-simple group $G$, embedded in a linear space.

Consider the rectangular lattice from section 2.1 and denote the lattice spacing by $h$. As usual, we denote the values of the field $\phi$ on the lattice points by $\phi_{i, j}$. We discretize the reduced partial derivatives $\phi^{-1} \phi_{x}$ and $\phi^{-1} \phi_{y}$ by writing them as follows:

$$
\phi^{-1} \phi_{x} \approx \frac{1}{h} \phi_{i+1, j}^{T}\left(\phi_{i+1, j}-\phi_{i, j}\right) \quad \text { and } \quad \phi^{-1} \phi_{y} \approx \frac{1}{h} \phi_{i, j+1}^{T}\left(\phi_{i, j+1}-\phi_{i, j}\right),
$$


where $\phi_{i, j}^{T}$ is the transpose of the matrix $\phi_{i, j}$. Substituting this into (20) yields the following discrete Lagrangian (up to an unimportant constant):

$$
\mathcal{L}_{d}=-\frac{1}{h^{2}} \operatorname{tr}\left(\phi_{i, j}^{T} \phi_{i+1, j}\right)-\frac{1}{h^{2}} \operatorname{tr}\left(\phi_{i, j}^{T} \phi_{i, j+1}\right) .
$$

In order to ensure that $\phi_{i, j} \in S O(n)$, we need to impose the constraint that $\phi_{i, j}^{T} \phi_{i, j}=I$. We are thus led to consider the following action:

$$
S(\phi)=\sum_{i, j}\left(\operatorname{tr}\left(\phi_{i, j}^{T} \phi_{i+1, j}\right)+\operatorname{tr}\left(\phi_{i, j}^{T} \phi_{i, j+1}\right)-\frac{1}{2} \operatorname{tr}\left(\Lambda_{i, j}\left(\phi_{i, j}^{T} \phi_{i, j}-I\right)\right)\right),
$$

where we have rescaled the Lagrange multipliers $\Lambda_{i, j}$ to get rid of the factor $-1 / h^{2}$. Note that $\Lambda_{i, j}$ is a symmetric matrix.

The field equations are obtained by demanding that $S$ be stationary under arbitrary variations; they are given by

$$
\phi_{i+1, j}+\phi_{i-1, j}+\phi_{i, j+1}+\phi_{i, j-1}=\phi_{i, j} \Lambda_{i, j}
$$

We multiply these equations by $\phi_{i, j}^{T}$ from the right, and use the fact that $\phi_{i, j} \Lambda_{i, j} \phi_{i, j}^{T}$ is a symmetric matrix in order to get rid of the Lagrange multipliers:

$$
\begin{aligned}
& \phi_{i+1, j} \phi_{i, j}^{T}+\phi_{i, j+1} \phi_{i, j}^{T}+\phi_{i-1, j} \phi_{i, j}^{T}+\phi_{i, j-1} \phi_{i, j}^{T}= \\
& \phi_{i, j} \phi_{i+1, j}^{T}+\phi_{i, j} \phi_{i, j+1}^{T}+\phi_{i, j} \phi_{i-1, j}^{T}+\phi_{i, j} \phi_{i, j-1}^{T} .
\end{aligned}
$$

By introducing the following quantities,

$$
m_{i+1, j}=\phi_{i+1, j} \phi_{i, j}^{T}-\phi_{i, j} \phi_{i+1, j}^{T} \quad \text { and } \quad n_{i, j+1}=\phi_{i, j+1} \phi_{i, j}^{T}-\phi_{i, j} \phi_{i, j+1}^{T},
$$

the field equations can be rewritten as the following set of conservation laws:

$$
m_{i+1, j}+n_{i, j+1}=m_{i, j}+n_{i, j} .
$$

Finally, let us introduce the discrete momenta $M_{i, j}$ and $N_{i, j}$, defined as

$$
M_{i, j}=\phi_{i-1, j}^{T} m_{i, j} \phi_{i-1, j} \quad \text { and } \quad N_{i, j}=\phi_{i, j-1}^{T} n_{i, j} \phi_{i, j-1} .
$$

The field equations governing the behaviour of these quantities are then easily determined to be, on the one hand

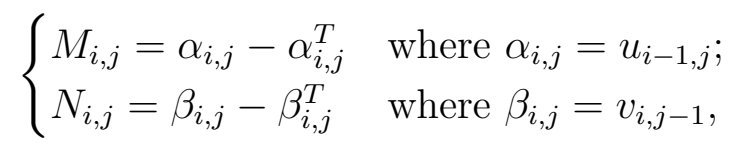

as well as, on the other hand, the counterpart of (23):

$$
M_{i+1, j}+N_{i, j+1}=\operatorname{Ad}_{\alpha_{i, j}^{T}} M_{i, j}+\operatorname{Ad}_{\beta_{i, j}^{T}} N_{i, j} .
$$

The similarities with the Moser-Veselov equations for the discrete rigid body are obvious (compare with equation (4) in [17]). 
Remark 5.1. It is now straightforward to see the equivalence between the MoserVeselov and the Euler-Poincaré equations. Indeed, starting from the reduced Lagrangian $l$, put

$$
M_{i+1, j}=R_{u_{i, j}}^{*} \mathrm{~d} l\left(\cdot, v_{i, j}\right) \quad \text { and } \quad N_{i, j+1}=R_{v_{i, j}}^{*} \mathrm{~d} l\left(u_{i, j}, \cdot\right),
$$

which can be interpreted as a discrete Legendre transformation [20]. Furthermore, put $\alpha_{i, j}=u_{i-1, j}$ and $\beta_{i, j}=v_{i, j-1}$. The Euler-Poincaré equations (요) then reduce to the Moser-Veselov equations derived above.

\subsection{Relation with the Euler-Poincaré equations}

Instead of deriving the Euler-Poincaré equations from the Moser-Veselov equations, we can also start directly from the discrete action sum (21) and proceed as in section 3 .

The unreduced field equations are given by (22). We now multiply these equations from the left (rather than from the right as in the derivation of the Moser-Veselov equations) to obtain the following set of discrete Euler-Poincaré equations:

$$
u_{i, j}+v_{i, j}+u_{i-1, j}^{T}+v_{i, j-1}^{T}=\Lambda_{i, j},
$$

together with the integrability condition

$$
u_{i, j} v_{i+1, j} u_{i, j+1}^{-1} v_{i, j}^{-1}=e .
$$

Using then the symmetry of $\Lambda_{i, j}$, we eliminate the multipliers $\Lambda_{i, j}$ to arrive at the following expression:

$$
u_{i, j}+v_{i, j}-u_{i-1, j}-v_{i, j-1}=u_{i, j}^{T}+v_{i, j}^{T}-u_{i-1, j}^{T}-v_{i, j-1}^{T} .
$$

If we view $\varphi$ as a $\mathfrak{g l}(n)$-valued discrete one-form in the sense of \$2 2.2, then the EulerPoincaré equations can be conveniently expressed using the discrete codifferential:

$$
\delta \varphi=(\delta \varphi)^{T},
$$

or $\llbracket \delta \varphi \rrbracket=0$, where $\llbracket \cdot \rrbracket$ denotes the antisymmetric part of a matrix: $\llbracket A \rrbracket=\frac{1}{2}\left(A-A^{T}\right)$. Many authors (see, for instance, [23, 5]) express the continuum equations for harmonic mappings taking values in a Lie group $G$ with bi-invariant metric as $\mathrm{d} \alpha=0=\delta \alpha$, where $\alpha$ is a $\mathfrak{g}$-valued one-form on the base space. Equations (27) and (28) are the discrete counterpart of these continuum equations.

\section{Generalizations}

\subsection{Field theories on non-trivial base spaces}

Up until now, we considered discretizations of field theories with two independent variables, i.e. where the base space is $\mathbb{R}^{2}$. From the beginning of the paper, we discretized 
$\mathbb{R}^{2}$ by considering the square mesh $(V, E, F)$ in $\mathbb{R}^{2}$ whose vertices have integer coordinates. Other discretisations of $\mathbb{R}^{2}$ can be introduced as in $[20$ ] by noting that $(V, E, F)$ is a planar graph; the theory of this paper can be readily generalized to the case of arbitrary planar graphs.

Secondly, the generalization to an arbitrary base space $X$, not necessarily $\mathbb{R}^{2}$, is straightforward under the assumption that a simplicial complex can be embedded in $X$. Let us now briefly discuss this case.

As before, unreduced and reduced fields are maps associating a group element to each 0-dimensional and 1-dimensional simplex, respectively. Other generalizations are also possible, for instance where one considers fields that associate group elements to higherdimensional simplices, but these types of fields do not arise in Euler-Poincaré reduction and cannot be treated with the Lie groupoid framework of section 6.2. For an introduction to such field theories, see [22].

The theory of discrete differential forms starting from a simplicial complex and its dual is developed to great detail in [8, 10] for spaces of arbitrary dimension and topology. To study discrete Lagrangian field theories, one now has to focus on the Cartesian products $G^{\times(n+1)}$ and $G^{\times n}$, where $n+1$ is the dimension of the base space. The usual procedures of discrete Lagrangian field theories and discrete reduction of the previous sections carry through to this case without significant changes.

A significant change occurs when the base space is not simply connected. In that case, proposition 2.6] is no longer valid. Consequently, a reduced field then no longer induces a uniquely determined unreduced field. This is similar to the continuous case; see [5].

\subsection{Discrete field theories on Lie groupoids}

For the better part of this paper, we have considered discrete field theories associating group elements to either the vertices (unreduced fields), or to the edges of the mesh (reduced fields). It turns out that both are special cases of discrete field theories taking values in Lie groupoids. This point of view was introduced in [20], to which we refer for a detailed overview. In this section, we will briefly show how Lie groupoid field theories provide a unified framework for Euler-Poincaré reduction. We will focus mostly on a Lie groupoid version of theorem 3.4, but note that other constructions, such as the Noether theorem and the Poincaré forms of section 4, also have their counterpart in the Lie groupoid framework.

For more information on Lie groupoids, as well as their role in discrete mechanics, the reader is referred to [13, 21, 14].

A Lie groupoid is a set $G$ with a partial multiplication $m$, a subset $Q$ of $G$ whose elements are called identities, two submersions $\alpha, \beta: G \rightarrow Q$ (called source and target maps respectively), which both equal the identity on $Q$, and an inversion mapping $i: G \rightarrow G$. A pair of Lie groupoid elements $(g, h)$ is said to be composable if the 
multiplication $m(g, h)$ is defined; the set of composable pairs will be denoted by $G_{2}$. We will denote the multiplication $m(g, h)$ by $g h$ and the inversion $i(g)$ by $g^{-1}$. In addition, these data must satisfy the following properties, for all $g, h, k \in G$ :

1. the pair $(g, h)$ is composable if and only if $\beta(g)=\alpha(h)$, and then $\alpha(g h)=\alpha(g)$ and $\beta(g h)=\beta(h)$;

2. if either $(g h) k$ or $g(h k)$ exists, then both do, and they are equal;

3. $\alpha(g)$ and $\beta(g)$ satisfy $\alpha(g) g=g$ and $g \beta(g)=g$;

4. the inversion satisfies $g^{-1} g=\beta(g)$ and $g g^{-1}=\alpha(g)$.

A Lie group $G$ can be considered as a Lie groupoid over a singleton $\{e\}$ : the source and target maps $\alpha$ and $\beta$ map any group element onto $e$ and the multiplication is defined everywhere.

Another example is the pair groupoid $Q \times Q$, where $Q$ is a manifold. The pair groupoid is a Lie groupoid over $Q$, and the source and target mappings are defined as follows: $\alpha\left(q_{0}, q_{1}\right)=q_{0}$, and $\beta\left(q_{0}, q_{1}\right)=q_{1}$. The multiplication of composable elements is then given by $\left(q_{0}, q_{1}\right)\left(q_{1}, q_{2}\right)=\left(q_{0}, q_{2}\right)$. The set of units in $Q \times Q$ is $\{(q, q): q \in Q\}$.

A groupoid morphism is a pair of maps $\phi: G \rightarrow G^{\prime}$ and $f: Q \rightarrow Q^{\prime}$ satisfying $\alpha^{\prime} \circ \phi=f \circ \alpha, \beta^{\prime} \circ \phi=f \circ \beta$ and such that $\phi(g h)=\phi(g) \phi(h)$ whenever $(g, h)$ is composable. Note that $(\phi(g), \phi(h))$ is a composable pair whenever $(g, h)$ is composable.

\subsubsection{Discrete fields}

The set of edges $E$ is a subset of the pair groupoid $V \times V$, but it is not a groupoid in itself because the multiplication of two elements of $E$ is not necessarily again an element of $E$. Nevertheless, it turns out that much can be gained from considering $E$ as a "local groupoid" in the sense of [20], rather than just as a subset of $V \times V$. By a "local groupoid", we mean here that, even if the multiplication is not defined in $E$, one can still define composable edges as pairs of edges $\mathfrak{e}_{1}, \mathfrak{e}_{2}$, such that $\beta\left(\mathfrak{e}_{2}\right)=\alpha\left(\mathfrak{e}_{1}\right)$, where $\alpha\left(x_{0}, x_{1}\right)=x_{0}$ and $\beta\left(x_{0}, x_{1}\right)=x_{1}$, i.e. $\alpha$ and $\beta$ are the usual source and target mappings of the pair groupoid $V \times V$, restricted to $E$. Hence, $E$ is a groupoid in all aspects but one, the multiplication.

With the conventions introduced above, we have for $\mathfrak{e}=\left(x_{0}, x_{1}\right)$ that $\mathfrak{e}^{-1}=\left(x_{1}, x_{0}\right)$. In addition to the mesh $(V, E)$ in $\mathbb{R}^{2}$, we now consider an arbitrary Lie groupoid $\Gamma$ over a manifold $Q$. The idea is to define discrete fields as mappings from the "local groupoid" $E$ to the Lie groupoid $\Gamma$. In particular, if $\varphi$ is a discrete field, then $\varphi$ maps composable edges in $E$ to composable elements in $\Gamma$, and, secondly, if $\mathfrak{e}$ is any edge in $E$, then $\varphi\left(\mathfrak{e}^{-1}\right)=\varphi(\mathfrak{e})^{-1}$. This is worked out in more detail in the following definition:

Definition 6.1. A discrete field is a pair $\varphi=\left(\varphi_{(0)}, \varphi_{(1)}\right)$, where $\varphi_{(0)}$ is a map from $V$ to $Q$ and $\varphi_{(1)}$ is a map from $E$ to $\Gamma$ such that 
1. $\alpha\left(\varphi_{(1)}(x, y)\right)=\varphi_{(0)}(x)$ and $\beta\left(\varphi_{(1)}(x, y)\right)=\varphi_{(0)}(y)$;

2. for each $(x, y) \in E, \varphi_{(1)}(y, x)=\left[\varphi_{(1)}(x, y)\right]^{-1}$;

3. for each $x \in V, \varphi_{(1)}(x, x)=\varphi_{(0)}(x) \in Q$.

Even though we are working with an object $E$ which is not quite a groupoid, it turns out that any discrete field $\varphi: E \rightarrow \Gamma$ may be extended to an actual groupoid morphism from $V \times V$ to $\Gamma$. This can be shown quite easily; for a proof, we refer to proposition 7 in [20]. Note that proposition [2.6] is a special case of this extension property.

Example 6.2. Consider a discrete field $\left(\varphi_{(0)}, \varphi_{(1)}\right)$ taking values in the pair groupoid $Q \times Q$. Because of the conditions in definition 6.1 the map $\varphi_{(1)}: V \times V \rightarrow Q \times Q$, is equal to $\varphi_{(0)} \times \varphi_{(0)}$. In this case, the discrete field is completely specified once we are given $\varphi_{(0)}: V \rightarrow Q$. For $Q=G$, this corresponds to the class of unreduced fields studied in section 3 .

Example 6.3. Consider now the case where the Lie groupoid $\Gamma$ is a Lie group $G$ and let $\left(\varphi_{(0)}, \varphi_{(1)}\right)$ be a discrete field. As $\varphi_{(0)}(x)=e$ for all $x \in V$, a discrete field can be identified with a map $\varphi_{(1)}: V \times V \rightarrow G$. As in section 3.3. this is a flat discrete connection, or, equivalently, a reduced field.

\subsubsection{Reduction}

The previous two examples show that the Lie groupoid framework encompasses both the case of unreduced and reduced fields (when $\Gamma=G \times G$ or $\Gamma=G$, respectively). The following theorem shows that the Euler-Poincaré reduction of one Lie groupoid field theory yields another one:

Theorem 6.4 (see [20]). Let $\Gamma^{\prime}$ be a Lie groupoid over a manifold $Q^{\prime}$ and consider a morphism $(\Phi, f):(\Gamma, Q) \rightarrow\left(\Gamma^{\prime}, Q^{\prime}\right)$. Furthermore, let $L^{\prime}: \mathbb{G}^{\prime k} \rightarrow \mathbb{R}$ be a Lagrangian on $\mathbb{G}^{\prime k}$ and consider the induced Lagrangian $L=L^{\prime} \circ \Psi$ on $\mathbb{G}^{k}$, where $\Psi: \mathbb{G}^{k} \rightarrow \mathbb{G}^{\prime k}$ is the map associated to $\Phi$.

A morphism $\phi: V \times V \rightarrow \Gamma$ will satisfy the discrete field equations for $L$ if the induced morphism $\Phi \circ \phi: V \times V \rightarrow \Gamma^{\prime}$ satisfies the discrete field equations for $L^{\prime}$.

Lemma 6.5. If the morphism $(\Phi, f)$ in theorem 6.4 is a submersion, then a discrete field $\phi: V \rightarrow V \rightarrow \Gamma$ will satisfy the discrete field equations for $L$ if and only if the induced field $\Phi \circ \phi: V \times V \rightarrow \Gamma^{\prime}$ satisfies the field equations for $L^{\prime}$.

Proof: The proof follows that of Corollary 4.7 in [14].

A few remarks are in order here. The manifold $\mathbb{G}^{k}$ consists of sequences of $k$ composable elements $\left(g_{1}, g_{2}, \cdots, g_{k}\right)$ in $\Gamma$, such that $g_{1} \cdot g_{2} \cdots g_{k}$ is a unit:

$$
\begin{gathered}
\mathbb{G}^{k}=\left\{\left(g_{1}, g_{2}, \ldots, g_{k}\right) \in G^{\times k}: \alpha\left(g_{i+1}\right)=\beta\left(g_{i}\right), \alpha\left(g_{1}\right)=\beta\left(g_{k}\right) \quad\right. \text { and } \\
\left.g_{1} \cdot g_{2} \cdots g_{k}=e_{\alpha\left(g_{1}\right)}\right\} .
\end{gathered}
$$


The manifold $\mathbb{G}^{k}$ is the manifold where the Lagrangian is defined, and as such it is the generalization to the case of Lie groupoids of the Cartesian products $G \times G \times G$ and $G \times G$ used in section 3.1. It is straightforward to check that, for $k=3, \mathbb{G}^{3}=G \times G \times G$ when $\Gamma=G \times G$, and $\mathbb{G}^{3}=G \times G$ for $\Gamma=G$.

The map $\Phi: G \times G \rightarrow G$, introduced in section 3.1. and defined as $\Phi\left(g, g^{\prime}\right)=g^{-1} g^{\prime}$, is a Lie groupoid morphism. When we put $\Gamma=G \times G$ and $\Gamma^{\prime}=G$, and use $\Phi$ in theorem 6.4. we obtain the reduction theorem 3.4. In contrast to discrete mechanics, it is not very likely that a reconstruction theorem can be proved in the context of Lie groupoids; in the case of Lie groups, for instance, there is the obstruction of flatness.

\section{Concluding remarks}

In this paper, we have shown that the well-known concepts of symmetry and EulerPoincaré reduction can be extended by very modest means to the case of discrete field theories. It turned out that many of the constructions known from continuum field theories have a natural discrete counterpart. However, much remains to be done.

Throughout the text, we remarked that almost all of our constructions can be extended to the case of field theories taking values in an arbitrary Lie groupoid $\Gamma$. Such a framework might be useful in the case of general Lagrangian reduction. In addition, as we pointed out before, many of the definitions introduced here gain much in clarity when rephrased in this more general language.

As a second point of interest, we are also interested in the concept of multisymplectic-

ity. Because of their variational nature, it is to be expected that the Euler-Poincaré equations are multisymplectic in the sense of [16, 4]. However, as the focus of this article was on theoretical developments rather than on the construction of practical integration schemes, we have chosen to leave this topic for future work.

\section{Acknowledgements}

The author is a Research Assistant of the Research Foundation - Flanders (Belgium), whose financial support is gratefully acknowledged. I would like to thank Frans Cantrijn for useful discussions and a critical reading of this manuscript. Furthermore, I am grateful to an anonymous referee for valuable criticisms which significantly improved both the contents and the presentation of this paper. 


\section{References}

[1] A. I. Bobenko and Y. B. Suris, Discrete time Lagrangian mechanics on Lie groups, with an application to the Lagrange top, Comm. Math. Phys. 204 (1999), no. 1, $147-188$.

[2] A. I. Bobenko and Y. B. Suris, Discrete Lagrangian reduction, discrete EulerPoincaré equations, and semidirect products, Lett. Math. Phys. 49 (1999), no. 1, 79-93.

[3] T. J. Bridges, Multi-symplectic structures and wave propagation, Math. Proc. Cambridge Philos. Soc. 121 (1997), no. 1, 147-190.

[4] T. J. Bridges and S. Reich, Multi-symplectic integrators: numerical schemes for Hamiltonian PDEs that conserve symplecticity, Phys. Lett. A 284 (2001), no. 4-5, 184-193.

[5] M. Castrillón López, P. L. García Pérez, and T. S. Ratiu, Euler-Poincaré reduction on principal bundles, Lett. Math. Phys. 58 (2001), no. 2, 167-180.

[6] M. Castrillón López and T. S. Ratiu, Reduction in principal bundles: covariant Lagrange-Poincaré equations, Comm. Math. Phys. 236 (2003), no. 2, 223-250.

[7] M. de León, D. Martín de Diego, and A. Santamaría-Merino, Symmetries in classical field theory, Int. J. Geom. Meth. Mod. Phys. 1 (2004), no. 5, 651-710.

[8] M. Desbrun, A. N. Hirani, M. Leok, and J. E. Marsden, Discrete exterior calculus, preprint (2005), math.DG/0508341.

[9] A. Hatcher, Algebraic topology, Cambridge University Press, Cambridge, 2002, available electronically from http: //www . math. cornell. edu/ hatcher/AT/ATpage.html.

[10] A. N. Hirani, Discrete Exterior Calculus, Ph.D. thesis, California Institute of Technology, 2003, available electronically from http://www.cs.uiuc.edu/homes/hirani.

[11] S. Kobayashi and K. Nomizu, Foundations of differential geometry. Vol I, Interscience Publishers, John Wiley \& Sons, 1963.

[12] M. Leok, J. E. Marsden, and A. D. Weinstein, A discrete theory of connections on principal bundles, preprint, 2005, math.DG/0508338.

[13] K. C. H. Mackenzie, General theory of Lie groupoids and Lie algebroids, London Mathematical Society Lecture Note Series, vol. 213, Cambridge University Press, Cambridge, 2005. 
[14] J. C. Marrero, D. Martín de Diego, and E. Martínez, Discrete Lagrangian and Hamiltonian Mechanics on Lie Groupoids, Nonlinearity 19 (2006), no. 6, 13131348, math.DG/0506299.

[15] J. E. Marsden, S. Pekarsky, and S. Shkoller, Discrete Euler-Poincaré and LiePoisson equations, Nonlinearity 12 (1999), no. 6, 1647-1662.

[16] J. E. Marsden, G. W. Patrick, and S. Shkoller, Multisymplectic geometry, variational integrators, and nonlinear PDEs, Comm. Math. Phys. 199 (1998), no. 2, 351-395.

[17] J. Moser and A. Veselov, Discrete versions of some classical integrable systems and factorization of matrix polynomials, Comm. Math. Phys. 139 (1991), no. 2, 217-243.

[18] S. P. Novikov, Discrete connections and linear difference equations, Tr. Mat. Inst. Steklova 247 (2004), 186-201, math-ph/0303035.

[19] D. J. Saunders, The Geometry of Jet Bundles, London Mathematical Society Lecture Note Series, vol. 142, Cambridge University Press, 1989.

[20] J. Vankerschaver and F. Cantrijn, Discrete lagrangian field theories on Lie groupoids, J. Geom. Phys. 57 (2007), no. 2, 665-689, math-ph/0511080.

[21] A. Weinstein, Lagrangian mechanics and groupoids, Mechanics day (Waterloo, ON, 1992), Fields Inst. Commun., vol. 7, Amer. Math. Soc., Providence, RI, 1996, pp. 207-231.

[22] D. Wise, Lattice p-Form Electromagnetism and Chain Field Theory, preprint, gr-qc/0510033.

[23] J. C. Wood, Harmonic maps into symmetric spaces and integrable systems, Harmonic maps and integrable systems (Braunschweig), Aspects Math., E23, Vieweg, 1994, pp. 29-55. 